www.jmscr.igmpublication.org

Impact Factor (SJIF): 6.379

Index Copernicus Value: 71.58

ISSN (e)-2347-176x ISSN (p) 2455-0450

crossrefDOI: https://dx.doi.org/10.18535/jmscr/v6i7.109

Journal Of Medical Science And Clinical Research

IGM Publication

An Official Publication of IGM Publication

\title{
Mitral Leaflet Separation Index (MLSI): A Step Ahead to Assess Severity of Mitral Stenosis in Patients with Atrial Fibrillation
}

\author{
Authors \\ Dr Raj Kumar Verma ${ }^{1}$, Dr Mridul Chaturvedi ${ }^{2}$, Dr Maaz Farooqui ${ }^{3 *}$ \\ Dr Dinesh Pal Singh ${ }^{4}$ \\ ${ }^{1}$ Lecturer, P.G. Dept. of Medicine, S.N. Medical College, Agra (INDIA) \\ ${ }^{2}$ Professor, P.G. Department of medicine, S.N. Medical College, Agra, Uttar Pradesh, INDIA \\ ${ }^{3,4}$ Junior Resident, P.G. Dept. of Medicine, S.N. Medical College, Agra (INDIA) \\ *Corresponding Author \\ Dr Maaz Farooqui \\ Email: farooquimaaz@gmail.com,7055918618
}

\begin{abstract}
Objective: Evaluation of the severity of mitral stenosis using mitral leaflet separation index and its significance over planimetry and pressure half time method especially in patients with Atrial fibrillation.

Material and Methods: This study was a hospital based prospective study which was done in S.N Medical College Agra. It was conducted on the patients attending O.P.D and also on those admitted in PG Department of Medicine, S $N$ Medical College, Agra. It included both patients who were already diagnosed and also those who were diagnosed for the first time in our department. A total of 100 cases between 18-60 yrs of age were included in the study. Cases already diagnosed with mitral stenosis but have undergone valvuloplasty or with severe co-morbid illnesses or other underlying cardiac abnormalities like infective endocarditis, atrial septal defect, ventricular septal defect were excluded from the study. Severity of mitral stenosis was also assessed using Planimetry and Pressure Half Time

Results: The correlation coefficient between the mitral leaflet separation index and the mitral valve area as measured by planimetry is 0.962 and the coefficient of determination is 0.927 which shows a very high correlation between both the methods. The correlation coefficient between the mitral leaflet separation index and the mitral valve area as measured by planimetry is 0.9555 and the coefficient of determination is 0.913 which shows a very high correlation between both the methods. The correlation coefficient between the mitral leaflet separation index and the mitral valve area as measured by planimetry is 0.895 and the coefficient of determination is 0.802 which shows a very high correlation between both the methods. The correlation coefficient between the mitral leaflet separation index and the mitral valve area as measured by planimetry is 0.836 and the coefficient of determination is 0.7 which shows a very high correlation between both the methods.

Conclusion: MLS index is a reliable method which can be used to assess severity of Mitral stenosis, and it may also be used as surrogate to current methods of assessment but not as a substitute for other echo parameters. This index would also help when there is a inconsistency between severities of MS estimated by present methods, in the existence of atrial fibrillation and in the presence of mitral regurgitation or where it is not possible to perform MVA calculation by planimetry. Especially in patients with Atrial fibrilaton where pressure half time method is not reliable and planimetry is not able to assess the area accurately due to poor echo window, Mitral leaflet separation index can be used as relable method to assess the severity.

Keywords: Mitral Leaflet Seperation Index, severity of mitral stenosis, assessment of severity of mitral stenosis in presence of atrial fibrillation, measurement of MVA by planimetry, measurement of MVA by pressure half-time method.
\end{abstract}




\section{Introduction}

Rheumatic fever is the leading cause of mitral stenosis. ${ }^{[1]}$ Other less common etiologies of obstruction to left atrial outflow include congenital mitral valve stenosis, cor-triatriatum, mitral annular calcification with extension onto the leaflets, systemic lupus erythematosus, rheumatoid arthritis, left atrial myxoma, and infective endocarditis with large vegetations. ${ }^{[2,3]}$ However in developing countries like India rheumatic heart disease is the most common cause. Direct measurement of MVA by planimetry is accurate but is highly operator dependent and sometimes laborious. ${ }^{[4]}$ The reliability of the pressure half-time method is affected by changes in preload or left ventricular compliance. ${ }^{[5]} \mathrm{A}$ novel index Mitral leaflet separation index was advised to overcome the discrepancy shown by the conventional methods. This index would also help when there is a discrepancy between severities of MS estimated by existing methods, in the presence of atrial fibrillation and in the presence of mitral regurgitation. This new index could be a useful surrogate measure of the MVA. ${ }^{4}$

\section{Aims and Objective}

To evaluate the severity of mitral stenosis using mitral leaflet separation index and its significance over planimetry and pressure half time method especially in patients with Atrial fibrillation.

\section{Material and Methods}

The study was a hospital based prospective study and was done in S.N Medical College Agra. The study was done among the patients attending O.P.D and those admitted in PG Department of Medicine, S N Medical College, Agra including those who were already diagnosed and those who were diagnosed for the first time in our department and was done between March 2013 and August 2014. A total of 100 cases between 18-60 yrs of age were included in the study.Cases already diagnosed with mitral stenosis but have undergone valvuloplasty or with severe co-morbid illnesses or other underlying cardiac abnormalities like infective endocarditis, atrial septal defect, ventricular septal defect were excluded from the study. Severity of mitral stenosis was also assessed using Planimetry and Pressure Half Time.

Mitral leaflet separation index- was measured by measuring maximal separation between inner edges of leaflets on parasternal long axis view and apical 4 chamber view. These two parameters are then averaged to measure mitral leaflet separation index. ${ }^{6}$

MLSI = Maximal separation on PLAX + A4C 2

(MLSI= Mitral Leaflet Seperation Index; Plax = Parasternal long axis view; $A 4 C=4$ chamber view)

Maximal separation of inner edges of mitral leaf lets on parasternal long axis view and apical four chamber view is measured and then averaged.

\section{Observations}

Mitral valve area was calculated by using Planimetry and pressure half time methods and MLSI was calculated by using above mentioned formula. Correlation coefficient was evaluated between the area calculated by both methods and MLSI which has been shown below. 
Figure 1 - Correlation between MVA by planimetry and MLSI

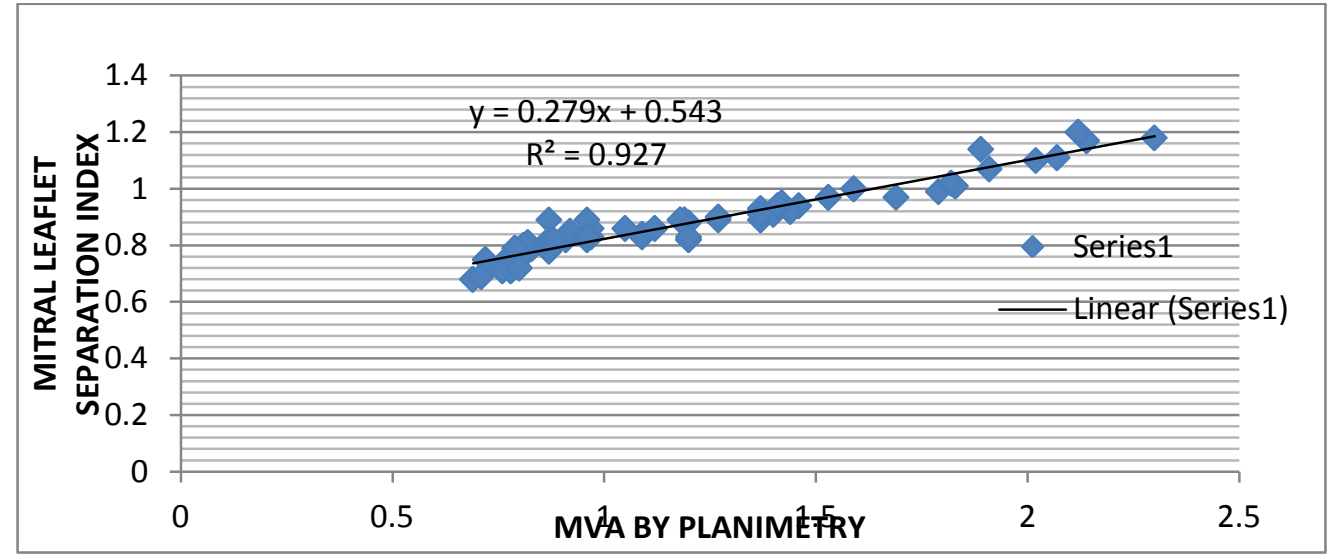

The correlation coefficient between the mitral coefficient of determination is 0.927 which leaflet separation index and the mitral valve shows a very high correlation between both the area as measured by planimetry is 0.962 and the methods.

Figure 2- Correlation between mitral valve area by pressure half time method and mitral leaflet separation index

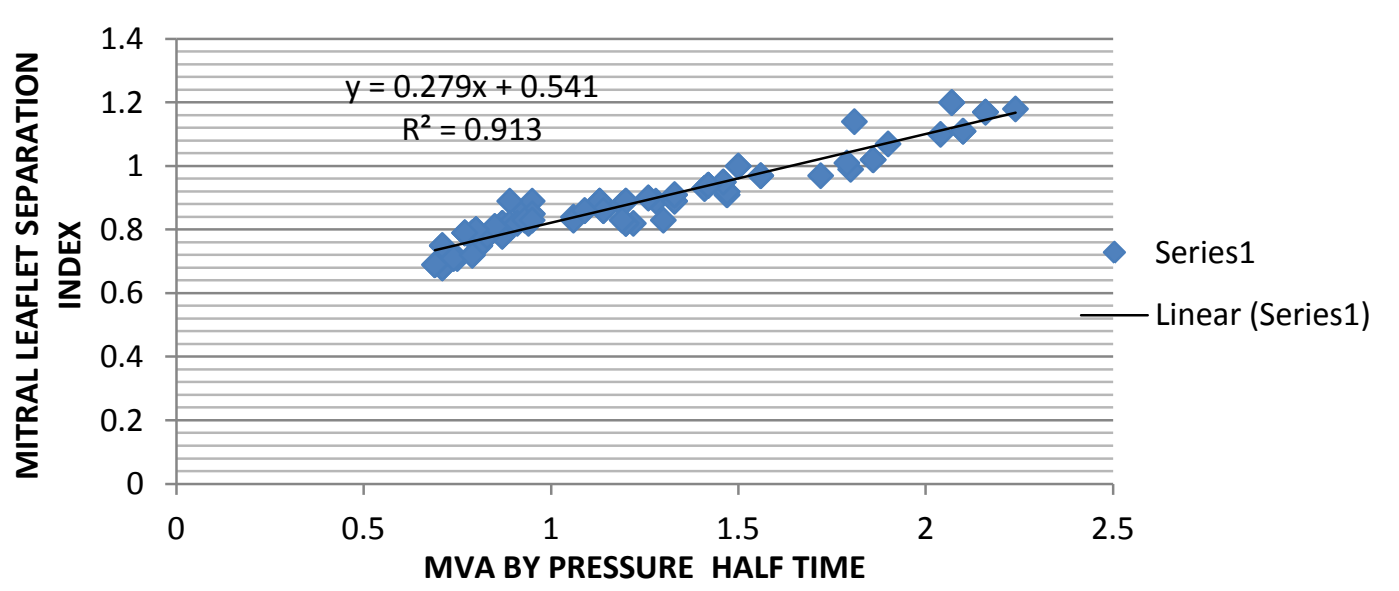

The correlation coefficient between the mitral leaflet separation index and the mitral valve area as measured by planimetry is 0.9555 and

the coefficient of determination is 0.913 which shows a very high correlation between both the methods.

Figure 3- Correlation between mitral leaflet separation index and mitral valve area by planimetry in patients with atrial fibrillation

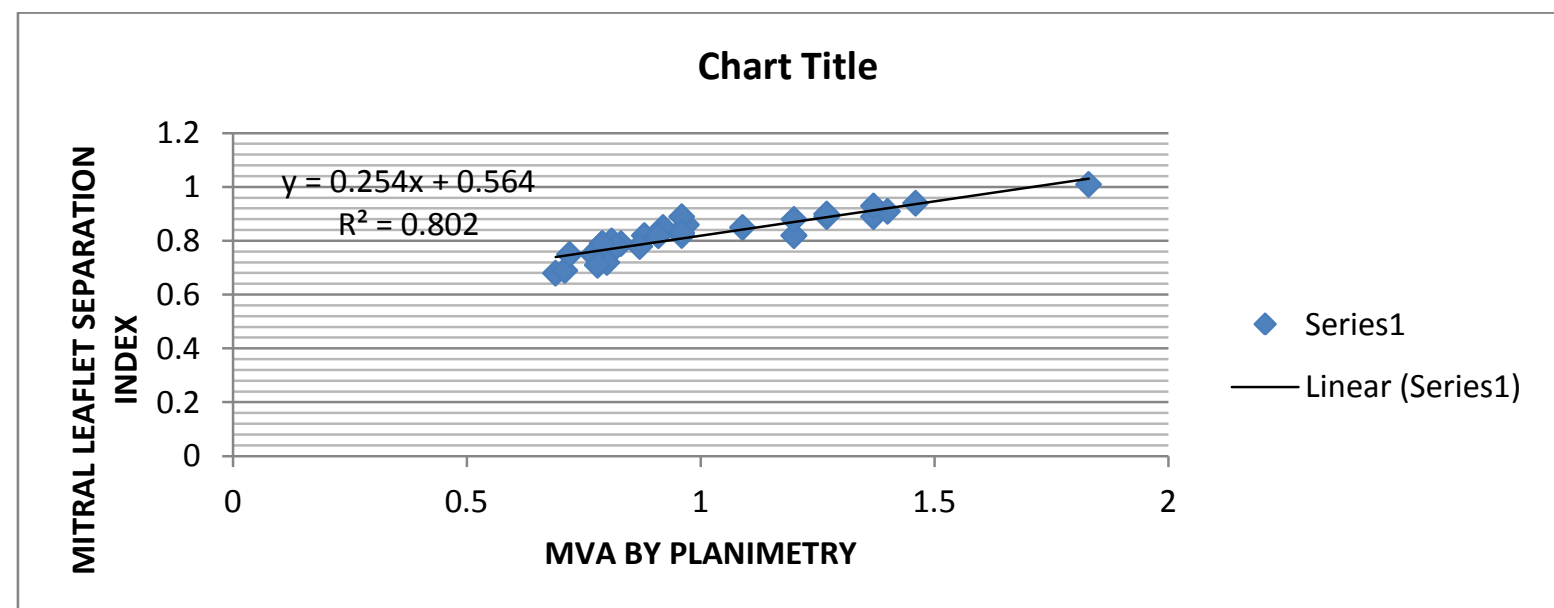


The correlation coefficient between the mitral leaflet separation index and the mitral valve area as measured by planimetry is 0.895 and the coefficient of determination is 0.802 which shows a very high correlation between both the methods.

Figure 4- Correlation between mitral leaflet separation index and mitral valve area by pressure half time method in patients with atrial fibrillation

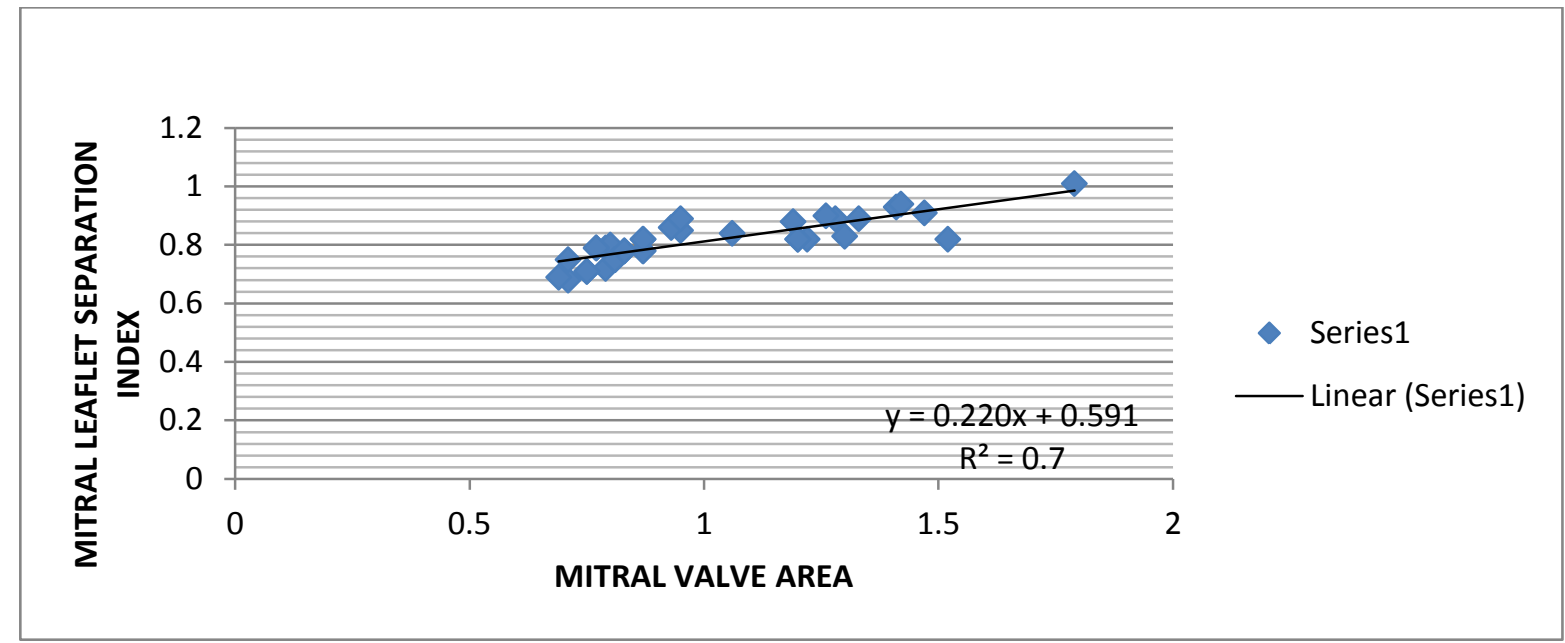

The correlation coefficient between the mitral leaflet separation index and the mitral valve area as measured by planimetry is 0.836 and the coefficient of determination is 0.7 which shows a very high correlation between both the methods.

Table 1 showing correlation coefficient between MVA by planimetry and PHT and MLSI in patient with and without atrial fibrillation

\begin{tabular}{|l|c|c|}
\hline & $\begin{array}{c}\text { In patient with no } \\
\text { Atrial fibrillation }\end{array}$ & $\begin{array}{c}\text { In patients with } \\
\text { Atrial Fibrillation }\end{array}$ \\
\hline Correlation between MVA by planimetry and MLSI & 0.962 & 0.895 \\
\hline Correlation between MVA by PHT and MLSI & 0.955 & 0.836 \\
\hline
\end{tabular}

\section{Discussion and Review of literature}

Above described observations clearly state that there is strong correlation between the area measured by Planimetry and Pressure half time and MLSI. However it is stronger between Planimetry and MLSI than Pressure Half time and MLSI especially in patients with atrial fibrillation. MLSI was first described in 1979 by Fisher et al and since then has been studied by various research groups and has been proven to be method of advantage in assessment of severity of mitral stenosis.

Fisher ML et al (1979) first described about assessment of severity of mitral stenosis by echocardiographic leaflet separation. Mitral valve area (MVA) determined at cardiac catheterization was compared with $\mathrm{M}$ mode echocardiographic measurements in 44 patients with mitral stenosis and no substantial mitral regurgitation. ${ }^{[2,6]}$ They concluded that despite statistically significant correlations, measurements of anterior leaflet motion, including rate of diastolic closure (EF slope) were not useful in predicting severity of stenosis. In contrast, maximal diastolic separation of anterior and posterior leaflets (SEP) was more closely correlated with MVA and appears to have some predictive value .Narrow separation was associated with severe mitral stenosis. Wide separation was associated with relatively mild stenosis. Intermediate values in 16 of 44 patients (36\%) were not of predictive value.Recognizing this restraint, measurement of maximal diastolic mitral leaflet separation from $M$ mode echocardiograms was projected as a simple and valuable method for assessing severity of mitral stenosis. ${ }^{[6]}$ 
Vimal Raj BS et al (2008) studied 150 consecutive patients with MS who underwent 2D echo. In each patient the severity of MS was assessed using 2D mitral valve area, pressure half time and MLS index. There were $22.7 \%$ patients with mild MS, $34.7 \%$ patients with moderate MS and $42.7 \%$ patients with severe MS. MLS index of $0.80 \mathrm{~cm}$ or less identified severe MS with $92 \%$ sensitivity and $92 \%$ specificity. MLS index of $>1.11 \mathrm{~cm}$ identified mild MS with $97 \%$ sensitivity and $97 \%$ specificity. Theyshowed that MLS demonstrate an excellent correlation with MVA by planimetry and the pressure half-time method. It is also significantly different for different grades of MS severity, demonstrating high discriminatory ability. It thus reliably differentiated patients with hemodynamically significant MS from those without. The MLS index showed very good correlation with MVA by planimetry in subgroup analysis of patients with AF. In presence of AF, at least five MLS readings in each view were taken and averaged.They concluded that the MLS index can be used as a screening method to semi quantify patients with mitral stenosis and should be added into yet another method to assess the severity. ${ }^{[7]}$ The criteria advised by vimal raj et al seems to be sensitive to identify the grade of severity of mitral stenosis. ${ }^{[5]}$

Holmin C, et al (2010) enrolled 90 consecutive patients, and concluded that no threshold value could predict a non-severe MS with both a sensitivity and a specificity greater than $80 \%$.A threshold value of $0.97 \mathrm{~cm}$ provided the best combination of sensitivity and specificity $(86 \%$ and $75 \%$, respectively). However, an MLS index of $1.2 \mathrm{~cm}$ or more provided a good specificity and positive predictive values for the diagnosis of nonsevere MS (85\% and 89\%, respectively) and an MLS index of less than $0.8 \mathrm{~cm}$ an excellent specificity and positive predictive value for severe MS (98\% and 96\%, respectively) and concluded that the MLS index cannot be considered as a substitute for MVA, however it can be used as a semiquantitative and complementary method for the integrative assessment of MS severity. ${ }^{[6]}$ Thus it remains accurate even in the presence of AF. It is a better indicator of MS severity than pressure gradient and can be used as a reliable tool to assess the severity of mitral stenosis in the presence of mitral regurgitation when mean gradient may overestimate the severity of mitral stenosis. Thus it is a better predictor of mitral stenosis severity in the presence of mitral regurgitation. ${ }^{[4]}$

Joby K. Thomas et al (2011) studied Mitral Leaflet Separation Index and concluded that MLS index is a reliable measure of MS severity, which can be used as a an easily obtainable adjunct and sometimes as a surrogate to current methods of assessment but not as a replacement for other echo parameters. This index was also found to be useful when there is a inconsistency between severities of MS estimated by existing methods, in the presence of atrial fibrillation and in the presence of mitral regurgitation. ${ }^{\text {[7] }}$

After review of literature it can be concluded that Mitral leaflet separation index worked as a good index to assess the severity in patients with mitral stenosis and was also found to be useful in patients where parasternal short axis view at the level of mitral valve was not possible due to altered cardiac anatomy or obese patients.

The main advantage of the MLSI index is its simplicity and ease of measurement in comparison with planimetry and Pressure half time. It provides a quick estimate of MS severity from standard 2D echocardiographic views without having to resort to tedious measurements as it is technically easy to obtain. The MLSI index could be especially useful in situations where there is disagreement between existing methods to the assessment of severity and hemodynamic significance of MS. MLSI index can thus be a useful supplement to the existing echo methods for assessment of MS.

MLSI index demonstrates an excellent correlation with MVA by planimetry and the pressure halftime method. It is also significantly different for different grades of MS severity, demonstrating high discriminatory ability. It thus reliably 
differentiated patients with hemodynamically significant MS from those without. The MLSI index showed very good correlation with MVA by planimetry in subgroup analysis of patients with AF. In presence of AF, at least five MLS readings in each view were taken and averaged. Thus it remains accurate even in the presence of AF. It is a better indicator of MS severity than pressure gradient and can be used as a reliable tool to assess the severity of mitral stenosis in the presence of mitral regurgitation when mean gradient may overestimate the severity of mitral stenosis. Thus it is a better predictor of mitral stenosis severity in the presence of mitral regurgitation. While calculating the MLSI it was also observed that in patients where the mitral valve area was too irregular due to calcification of the leaflets it correlated well with the symptoms of their symptoms and with pressure half time method or invasive methods.

While collecting the data it was found that MLSI in these patients correlated very well with their symptoms, with severe the symptoms, lesser was the value of Mitral leaflet separation index.Mitral leaflet separation index can be used as a measure for assessing the severity of mitral stenosis and it correlates very well with both planimetry $(0.962)$ and pressure half time method $(r=0.955)$. The value of correlation coefficient by both method is very near to 1 and hence shows that there is very high degree of correlation between both the variables. While assessing the severity of mitral stenosis in patients with atrial fibrillation MLSI correlates very well MVA by planimetry $(\mathrm{r}=0.895)$ and MVA by pressure half time ( $\mathrm{r}=$ 0.836), however correlation with MVA by planimetry is much higher than MVA by pressure half time method. It is due to the reason that pressure half time is not a perfect method to assess the mitral valve area as the pressure gradient between LA and LV changes with each cardiac cycle.

\section{Conclusion}

MLS index is a reliable method to assess severity of Mitral stenosis, and can be used as surrogate to current methods of assessment but not as a substitute for other echo parameters. This index would also help when there is ainconsistency between severities of MS estimated by present methods, in the existence of atrial fibrillation and in the presence of mitral regurgitation or where it is not possible to perform MVA calculation by planimetry.

Specially in patients with Atrial fibrilaton where pressure half time method is not reliable and planimetry is not able to assess the area accurately due to poor echo window, Mitral leaflet separation index can be used as relable method to assess the severity.

\section{References}

1. Marijon E, Ou P, Celermajer DS, et al: Prevalence of rheumatic heart disease detected by echocardiographic screening. N Engl J Med 2007; 357:470.

2. Assey ME, Usher BW, Carabello BA et al. The patient with valvular heart disease. In: Pepine CJ, Hill JA, Lambert CR, eds. Diagnostic and Therapeutic Cardiac Catheterization. Baltimore, MD: Williams \& Wilkins; 1989:471-507

3. Mitral Stenosis: Heart Valve Disorders: Merck Manual Home Edition". Retrieved 2009-03-14

4. Holmin C, Messika-Zeitoun D, Mezalek AT, Brochet E, Himbert $\quad D$, Iung B, Vahanian AMitral leaflet separation index: a new method for the evaluation of the severity of mitral stenosis? Usefulness before and after percutaneous mitral commissurotomy.

5. B. S. V. F. Raj, P. George, and V. J. Jose, "Mitral leaflet separation index-A simple novel index to assess the severity of mitral stenosis," IndianHeart Journal, vol. 60, no. 6 , pp. 563-566, 2008 
6. Fisher ML, Parisi AF, Plotnick GD. Assessment of severity of mitral stenosis by echocardiographic leaflet separation. Archives of Internal Medicine. 1979; 139(4): 402-404

7. Joby K. Thomas, T. M. Anoop, Gailin B. Sebastian, Kim George, and Raju George, "Mitral Leaflet Separation Index in Assessing the Severity of Mitral Stenosis," ISRN Cardiology, vol. 2011, Article ID 768097, 4 pages, 2011. doi:10.5402/2011/768097. 